\title{
Computing Call Dropping and Waiting Probabilities in LEO Satellite Systems for Voice Communications
}

\author{
Sebastià Galmés and Ramon Puigjaner \\ Dept. de Ciències Matemàtiques i Informàtica, Universitat de les Illes Balears \\ Cra. de Valldemossa, km. 7.5, 07122 Palma, Illes Balears, Spain \\ $\{$ dmisgo0, putxi\}@uib.es
}

\begin{abstract}
In this paper we propose and analyze a call management architecture for a cell in a satellite cellular network, where handoff arrivals are prioritized by using a number of guard channels and only new calls are temporarily buffered if necessary. The idea behind this is to avoid any extra delay during handoff procedures, in addition to the intrinsic round trip propagation time, since it might cause severe user dissatisfaction. The analysis is based on a Markov chain model of a single spot (cell) and yields exact expressions for the dropping and waiting probabilities of handoff and new arrivals respectively. Then, we derive an efficient algorithm to evaluate these probabilities, which is based on the well-known Erlang-B formula and its recursion. Finally, we use the expressions for the waiting and dropping probabilities to formulate and solve an optimization problem in the context of network planning. Numerical results are provided.
\end{abstract}

\section{Introduction}

Within the context of cellular wireless networks, several prioritized handoff procedures have been proposed in the literature, usually in one or both of the two following ways: by reserving a number of channels (called guard channels) for handoff connections, or by allocating some buffer space to them. Examples can be found in [1-6].

One way to support voice communications with mobile and personal terminals is by means of a cellular wireless network implemented as a constellation of LEO (Low Earth Orbit) satellites [7-11]. In essence, these systems are similar to the terrestrial ones, but they have to cope with the specific problems that arise when base stations are orbiting at a non-fixed apparent position around the earth. Particularly, there is also a need to manage the incoming handoff traffic into a cell, caused in this case either by the user mobility (Earth Fixed Coverage - EFC - systems) or by the satellite mobility (Satellite Fixed Coverage - SFC - systems).

More specifically, with regards to handoff management, there are some interesting suggestions in the literature. For instance, in [12-13] a buffer with a time deadline is used to store handoff connections that find all channels busy. Other works assume that a number of guard channels are reserved for handoff attempts [14]. In general, most of works rely on the combination of guard channels and buffer space as a way of prioritizing handoff connections. From our point of view, the presence of a buffer to temporally store handoff calls in a LEO system, adds an extra delay to the intrinsic 
round trip propagation time that users may not tolerate. Thus, to decrease the time consumption incurred by handoff procedures, we propose a model without buffering for this type of traffic, but with the necessary increment on the number of guard and/or common channels to keep the desired dropping probability. At the same time, since new calls are more tolerant to delays, we propose the use of a buffer exclusively for them, which in turn contributes to increase the total traffic carried by the cell.

The paper is organized as follows. In Section 2, we propose and analyze a Markov model for a single spot beam (cell) of a LEO satellite. In Section 3, we obtain exact analytical expressions for the dropping probability of handoff calls and the waiting probability of new calls. In Section 4, we discuss some computational aspects with regards to the formulas obtained in the previous section, and we provide an efficient algorithm to evaluate them. In Section 5, we test the proposed algorithm and obtain some numerical results. In Section 6, we propose a method to determine the feasible region of solutions for the common and guard channels, when the total number of channels is fixed by the satellite frequency plan, and certain bounds on the dropping and waiting probabilities are imposed. Finally, in Section 7, we draw the main conclusions and suggestions for further research.

\section{The Markov Model}

We consider the performance model of a single cell in a satellite cellular system. There is a Poisson arrival stream of new calls at the rate $\lambda_{1}$ and a Poisson arrival stream of handoff calls at the rate $\lambda_{2}$. Handoff calls may be caused either by the user mobility (EFC coverage) or by the satellite mobility (SFC coverage). There is a total amount of $\mathrm{N}$ channels (servers), where each one can hold a voice communication. An ongoing call (new or handoff) leaves a channel when its service is completed (call holding time) at a rate $\mu_{1}$, or when it is handed off, at a rate $\mu_{2}$. In order to prioritize handoff arrivals, a number of $g$ guard channels are reserved for them. The rest of channels $(c=N-g)$, called common or shared channels, are available to both arrival classes. This means that a new call is accepted only if $g+1$ or more channels are in idle state. Otherwise, the new call is queued on a FCFS buffer. We assume that the buffer has always space to allocate new calls, so from the mathematical point of view we model it as of infinite capacity. On the other hand, as we said before, there is no buffer space for handoff arrivals, in order to avoid extra delays.

Within the context of LEO satellite systems, the proposed model is quite general, since it can be adapted to represent EFC or SFC mechanisms, FCA or DCA schemes and both beam-to-beam and satellite-to-satellite handoffs.

The model so far described was already analyzed in other contexts in [15] and some references therein, but following different formulations and objectives. Particularly, in [15] the model was motivated by its possible application to cellular radio systems, as a way of increasing the total traffic supported by a cell while improving the perceived quality of service by users. This paper focused on the application of a novel methodology to obtain closed-form expressions for the steady-state probabilities. In the present paper, we re-formulate that analysis in the context of LEO satellite systems, by introducing a significant system parameter and by referring it to the well- 
known Erlang-B formula. The new formulation is simpler and facilitates the treatment of some multiobjective optimization problems that arise when planning the network.

According to the definitions given before, the global arrival rate is $\lambda=\lambda_{1}+\lambda_{2}$. Since any arrival (new or handoff) may leave the cell as a terminated call or after a handoff procedure, the total service rate is $\mu=\mu_{1}+\mu_{2}$.

Let $b$ be the number of busy channels and $S(t)$ the state of the system at time $t$. From the specification of the model, we propose the following state description:

$$
S(t)=\left\{\begin{array}{l}
0,1, \ldots, c-1, b<c \\
\{(c+i, j): i=0, \ldots, g ; j=0, \ldots, \infty\}, b \geq c
\end{array}\right.
$$

Here, variables $i$ and $j$ denote respectively the number of busy guard channels and the number of new calls present in the queue. The state transition diagram for the case $b<c$ is represented in Figure 1 .
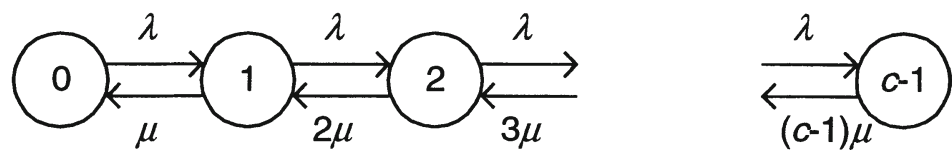

Fig. 1. State transition diagram for $b<c$

On the other hand, if $b \geq c$ the diagram takes the form of a meshed structure, as it is shown in Figure 2. Note that state $S(t)=c-1$ has been depicted again, since it links both the linear and meshed structures of the global state transition diagram. Since it in fact corresponds to an irreducible homogeneous continuous time Markov chain, an ergodic (stable) steady-state solution may exist. Then, if we assume that stability conditions are fulfilled, we can easily obtain the following solution for the steady-state probabilities $p_{k}$ associated with the linear diagram, where $A=\lambda \mu$ is the total traffic offered to the cell (in Erlangs):

$$
p_{k}=p_{0} \cdot \frac{A^{k}}{k !}, k=1, \ldots, c-1
$$

On the other hand, let $p_{c+i, j}$ be the steady-state probability of state $(c+i, j)$, with $i=0$, $\ldots, g$ and $j=0, \ldots, \infty$. Let also define $A_{1}=\lambda_{1} / \mu, A_{2}=\lambda_{2} / \mu$, so that $A=A_{1}+A_{2}$, and the following aggregate probabilities on a per-column basis:

$$
q_{c+i}=\sum_{j=0}^{\infty} p_{c+i, j}, i=0, \ldots, g
$$

Then, by following standard procedures based on applying balance equations to state aggregations [16], we can obtain the following result (see [15] for a more detailed derivation with another notation): 


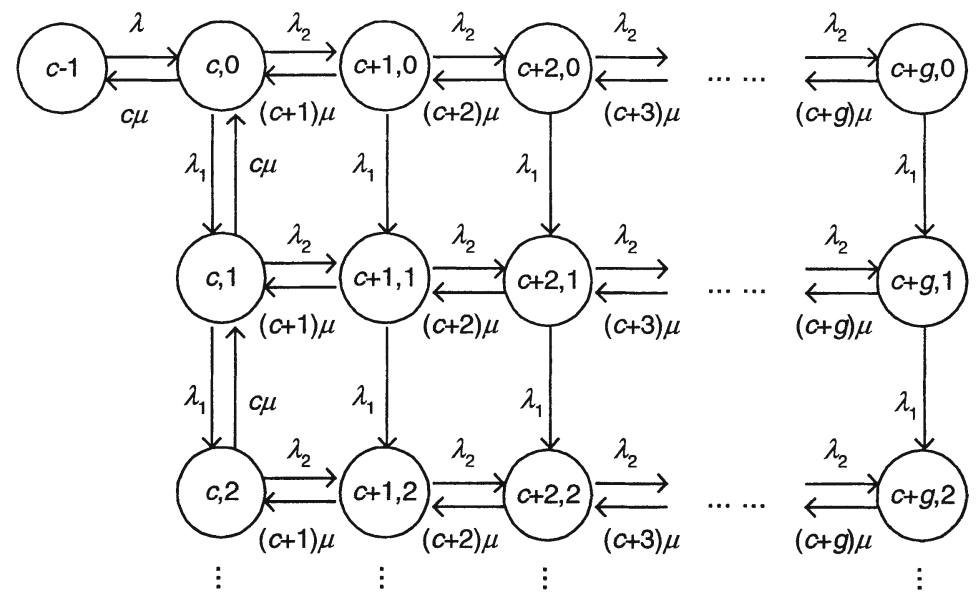

Fig. 2. State transition diagram for $b \geq \mathrm{c}$

$$
q_{c+i}=\frac{A_{2}^{i}}{(c+i) !} \frac{A^{c}}{1-\frac{A_{1} H}{c}} p_{0}, i=0, \ldots, g
$$

where

$$
H=\sum_{i=0}^{g} A_{2}^{i} \frac{c !}{(c+i) !}
$$

Here, $H \geq 1$ is an auxiliary parameter that it is directly proportional to the offered handoff traffic $\left(A_{2}\right)$ and the number of guard channels $(g)$. In fact, $H=1$ only if $A_{2}=0$ and/or $g=0$. Also, $H$ increases as the number of common channels decreases. Therefore, since all contributions to an increment on the value of $H$ are always associated with an increment of the buffer occupancy, we call $H$ the congestion parameter. On the other hand, the denominator in expression (4) reveals the unique stability condition for the system, which imposes an upper bound to $H$ :

$$
\frac{A_{1} H}{c}<1
$$

Finally, by applying the normalizing condition to all probabilities, we obtain $p_{0}$ :

$$
\sum_{k=0}^{c-1} p_{k}+\sum_{i=0}^{g} q_{c+i}=1 \Rightarrow p_{0}=\left(\sum_{k=0}^{c-1} \frac{A^{k}}{k !}+\frac{A^{c}}{c !} \cdot \frac{H}{1-\frac{A_{1} H}{c}}\right)^{-1}
$$




\section{Performance Measures}

From the steady-state aggregate probabilities, various performance measures can be obtained. Here, we are especially interested in the dropping probability of handoff calls and the waiting probability of new calls. These measures are directly related to the quality or grade of service perceived by users, and can be used as the basis for several optimization and design problems concerning the number of common and guard channels. An example of design problem is presented in Section 7.

\subsection{Dropping Probability of Handoff Calls}

The dropping probability of handoff calls $\left(P_{d}\right)$ is the probability that a handoff arrival finds all channels busy. Since handoff arrivals are Poisson, the PASTA argument holds and $P_{d}$ can be calculated as follows:

$$
P_{d}=P_{d}(c, g)=q_{c+g}=\frac{\frac{A^{c} \cdot A_{2}^{g}}{(c+g) !} \cdot \frac{1}{1-\frac{A_{1} \cdot H}{c}}}{\sum_{k=0}^{c-1} \frac{A^{k}}{k !}+\frac{A^{c}}{c !} \cdot \frac{H}{1-\frac{A_{1} \cdot H}{c}}}
$$

This expression becomes simpler when there are no guard channels in the system, in which case the congestion parameter is 1 , and even simpler when additionally the handoff traffic is zero $\left(A_{1}=A\right)$. In the latter case, the resulting model is nothing else but a pure $\mathrm{M} / \mathrm{M} / \mathrm{c}$ queue, and therefore the dropping probability becomes the Erlang-C formula.

\subsection{Waiting Probability of New Calls}

The waiting probability of new calls $\left(P_{w}\right)$ is the probability that an arriving new call cannot capture a channel immediately and has to wait in the queue. Obviously, this occurs whenever the number of busy channels is not less than the number of common channels:

$$
P_{w}=P_{w}(c, g)=\sum_{i=0}^{g} q_{c+i}=\frac{\frac{A^{c}}{c !} \cdot \frac{H}{1-\frac{A_{1} \cdot H}{c}}}{\sum_{k=0}^{c-1} \frac{A^{k}}{k !}+\frac{A^{c}}{c !} \cdot \frac{H}{1-\frac{A_{1} \cdot H}{c}}}
$$

Again, we can easily obtain particular simplifications of this result when there are no guard channels in the system or handoff traffic does not exist. For instance, if there are no guard channels, obviously the waiting and blocking probabilities coincide. If 
additionally the handoff traffic is zero, we again obtain the Erlang-C formula. From (8) and (9) we can derive the following relation between both probabilities:

$$
P_{w}(c, g)=P_{d}(c, g) \frac{H(c+g) !}{A_{2}^{g} \cdot c !}
$$

\section{Computational Aspects}

Usually the total number of voice channels available in the satellite is relatively large, leading to numerical difficulties in the evaluation of the performance measures. Thus, it is necessary to introduce recursive procedures in order to avoid overflow problems caused by the direct computation of large powers and factorials. Since both probabilities are related through expression (10), it is enough to find a recursion for one of them. Particularly, expression (9) for the waiting probability is simpler, and in fact it can be easily rewritten in terms of the standard Erlang-B formula as follows:

$$
P_{w}(c, g)=\frac{B(c, A)}{\left(\frac{1}{H}-\frac{A_{1}}{c}\right)+\left[1-\left(\frac{1}{H}-\frac{A_{1}}{c}\right)\right] B(c, A)}
$$

Then, the waiting probability can be calculated by using the well known recursion for the Erlang-B formula, traditionally used in the context of wired telephone networks [17]:

$$
B(c, A)=\frac{\frac{A}{c} B(c-1, A)}{1+\frac{A}{c} B(c-1, A)} ; B(0, A)=1.0
$$

On the other hand, expression (5) for the congestion parameter can be written in the following way for computational purposes:

$$
H=\sum_{i=g}^{0} h_{i}, h_{i}=h_{i-1} \frac{A_{2}}{c+i}, i=1, \ldots, g, h_{0}=1.0
$$

The idea behind (13) is to evaluate the congestion parameter by starting with the least significant term so as to reduce round-off errors.

Once the Erlang-B formula and the congestion parameter have been evaluated separately, the waiting probability can be easily derived from (11) and, next, the dropping probability through (10) following this practical implementation:

$$
P_{d}(c, g)=\frac{P_{w}(c, g)}{H} \prod_{i=1}^{g} \frac{A_{2}}{c+i}
$$

The following algorithm summarizes the proposed methodology: 


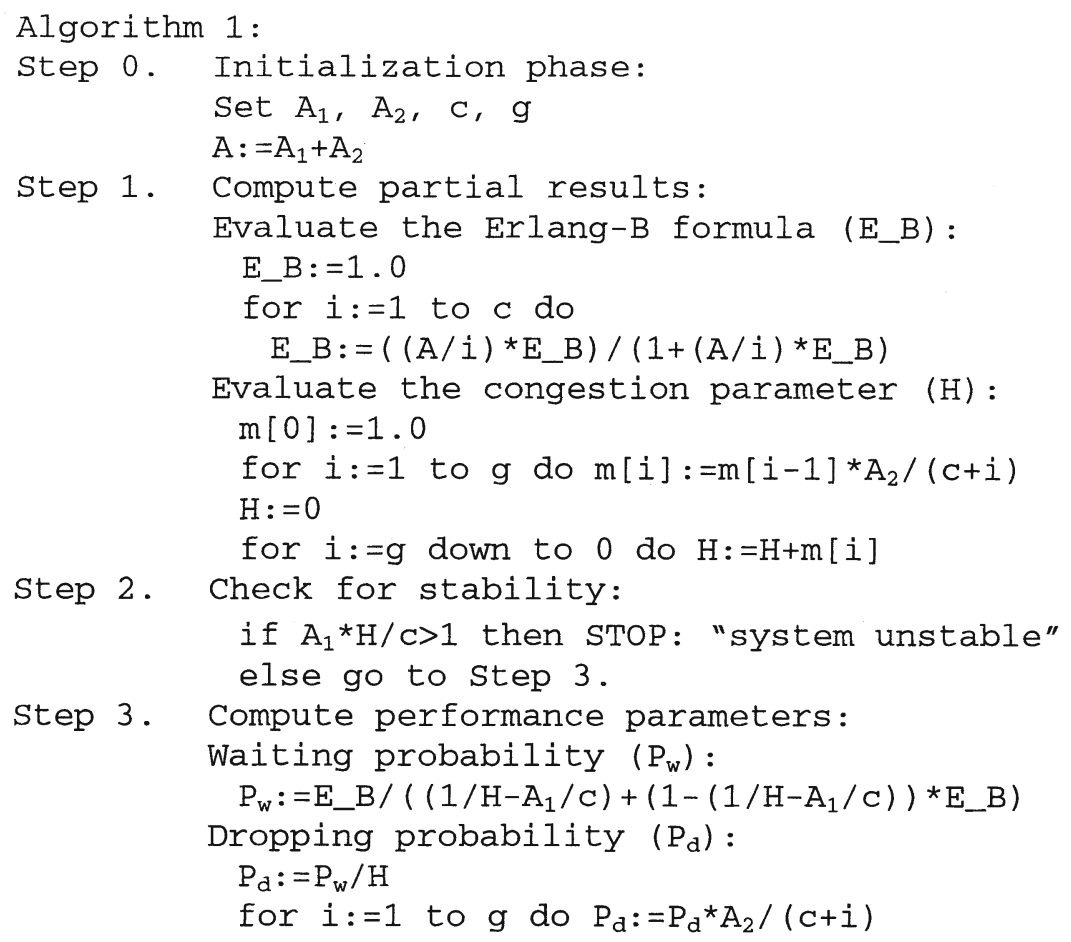

\section{Numerical Results}

In this section, we provide the results obtained from applying the proposed algorithm to different sets of input data. Particularly, we varied the number of shared channels (c) between 100 and 120, and the number of guard channels $(g)$ between 0 and 10 . For the remaining parameters, we assumed with no loss of generality $A_{1}=15$ and $A_{2}=90$.

As an intermediate result, Figure 3 shows the evolution of the congestion parameter $(H)$ as a function of $g$, for different values of $c$. For reasons of stability, the congestion parameter cannot achieve arbitrarily high values; in fact, condition (6) imposes the following upper bound to it:

$$
H<\frac{c}{A_{1}}
$$

Figure 4 plot the evolution of the waiting probability in terms of the number of common channels, with the number of guard channels as a parameter. As it is shown, the waiting probability decreases as the number of common channels increases, since more channels are available for new calls. On the other hand, an improvement of the service offered to handoff traffic, by increasing the amount of guard channels, causes more congestion to new calls. This is because more handoff calls are accepted in the system and it takes more time until all busy guard channels become idle. 

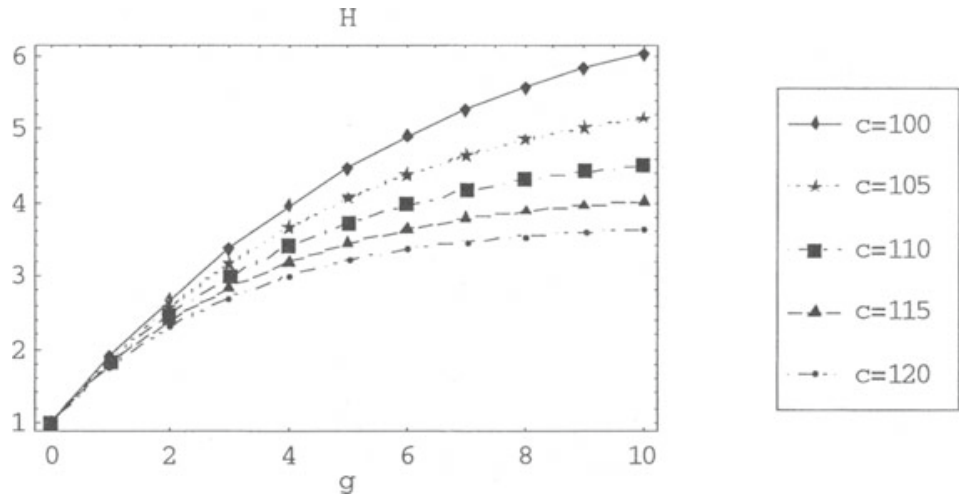

Fig. 3. The congestion parameter as a function of the number of guard channels, for different values of the number of common channels (the offered traffics are $A_{1}=15$ and $A_{2}=90$ )
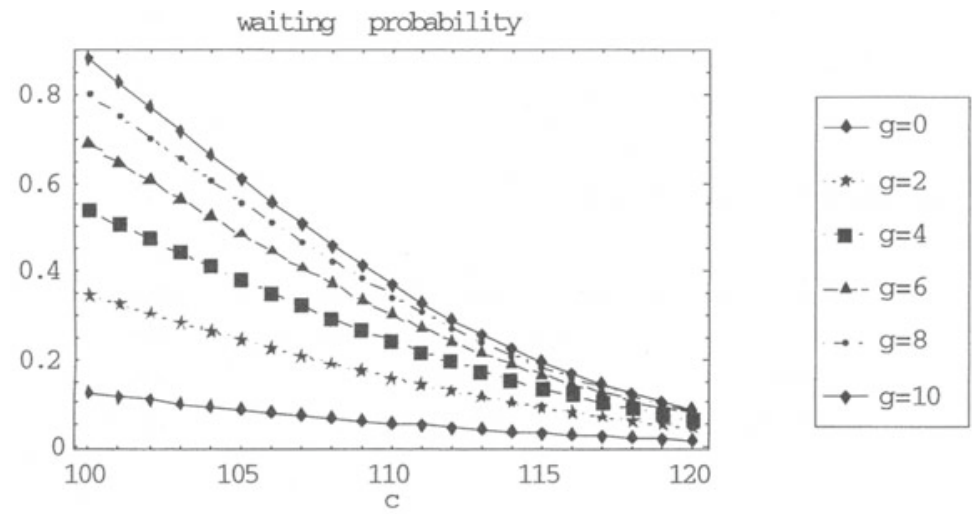

Fig. 4. The waiting probability as a function of both the common and guard channels $\left(A_{1}=15\right.$ and $A_{2}=90$ )

With regards to Figure 5, we see that an increment on the number of any type of channels always causes a benefit to handoff traffic.

Also, we have conducted some experiments to compare the buffered case studied in the present paper with the unbuffered case analyzed in [2]. In general, we have observed that for given values of the number of common and guard channels, the unbuffered case always behaves better than the buffered one with regards to dropping probability. This is because more new calls are accepted into the system when a buffer is present, in detriment of the handoff traffic (take into account that both traffic classes compete for common channels). Thus, in order to keep the quality of service offered to handoff traffic, the buffered configuration requires a larger number of guard and/or 
common channels. However, when this requirement is fulfilled, the buffered configuration supports a larger value of total carried traffic than the unbuffered one.

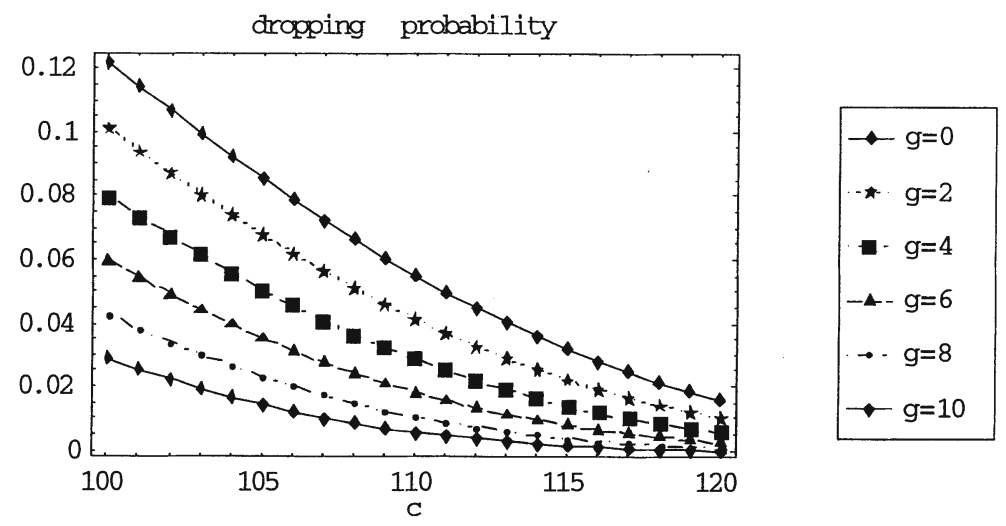

Fig. 5. The dropping probability as a function of both the common and guard channels $\left(A_{1}=15\right.$ and $A_{2}=90$ )

\section{Optimization Problem}

A typical problem is when the total number of channels per cell is fixed by the operator, and we want to explore the combinations of the numbers of common and guard channels that allow for fulfilling prefixed performance requirements in terms of the waiting and dropping probabilities. Depending upon the number of channels available or the restrictions imposed to those probabilities, the problem may not have a solution. However, if it does have it, it usually takes the form of a feasible region expressed as an interval [cmin, cmax] for the number of common channels, or as [gmin, gmax] for the number of guard channels (recall that $N=c+g$ is now fixed).

One way of facing this problem is by determining the feasible region for one performance measure, and then intersecting it with the feasible region of the other one. Since the total amount of channels is prefixed by the experiment, the numbers of both common and guard channels will follow opposite evolutions. Then, under this hypothesis, the behavior of the waiting probability becomes easier to predict than that of the dropping probability, as we can deduce from Figures 4 and 5. More formally, we can check the monotonic properties of the waiting probability by reformulating expression (9) as follows:

$$
P_{w}(c, g)=\frac{1}{1+\left(\frac{1}{H}-\frac{A_{1}}{c}\right)\left[\frac{1}{B(c, A)}-1\right]}
$$


Since both the congestion parameter and the Erlang-B formula decrease as the number of common channels increases, the waiting probability also decreases. On the other hand, if the number of guard channels increases, the congestion parameter and consequently the waiting probability also increase.

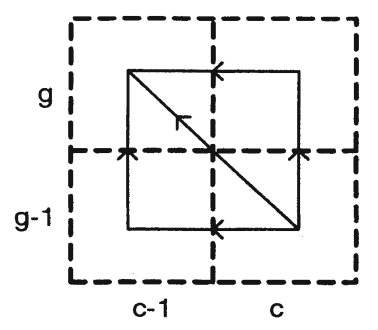

Fig. 6. Monotonic properties of the waiting probability

Figure 6 summarizes the monotonic properties of the waiting probability, and explains its adequateness to the design problem formulated above. Thus, we first investigate the feasible region of the waiting probability, and then evaluate both probabilities at each point belonging to it (on a point-per-point basis). This will provide the final feasible region, expressed as the interval [ $\mathrm{cmin}, \mathrm{cmax}$ ] mentioned before.

Suppose the total number of channels $N$ is fixed (by the operator), and we want to check the combinations of values of $c$ and $g$ such that $P_{w}(c, g)<P_{w 0}$ and $P_{d}(c, g)<P_{d 0}$, where $P_{w 0}$ and $P_{d 0}$ are certain given bounds. Algorithm 2 proposed below is designed to efficiently determine the minimum value of the number of common channels (cmin) necessary for the waiting probability to fulfill its requirement:

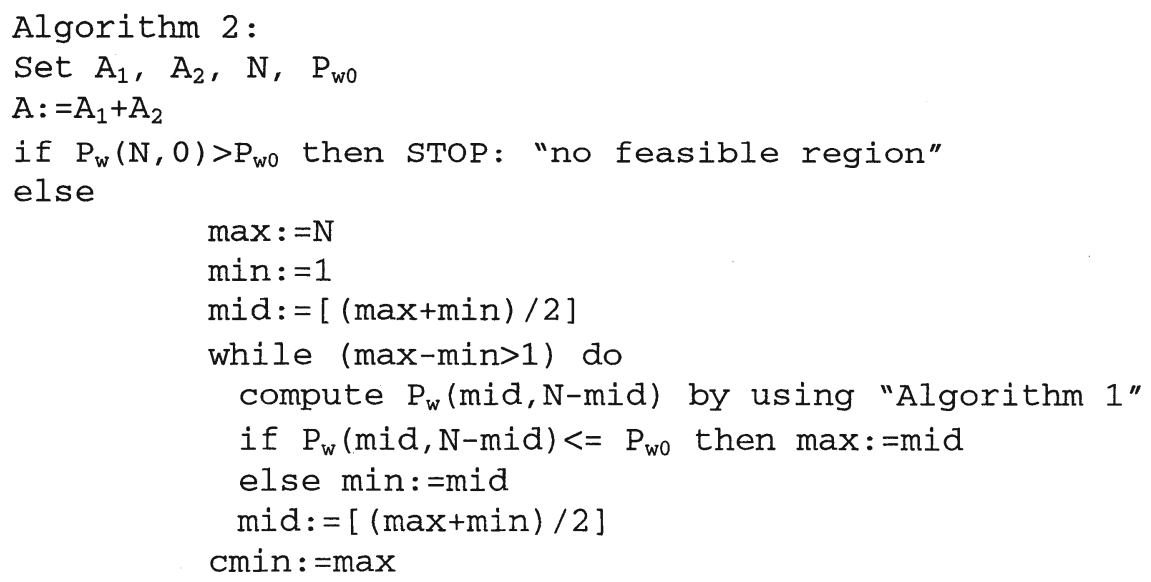

Note that the algorithm first checks if the requirement is satisfied at the extreme point $c=N$ and $g=0$. Because of the monotonic properties of the waiting probability described in Figure 6, if the test fails at this point, then there is no possible feasible region and the algorithm can conclude. Finally, the third algorithm evaluates both probabilities for $c \in[\mathrm{cmin}, N]$, taking into account that the number of guard channels must satisfy the condition $g=N-c$ : 


\section{Algorithm 3 : \\ for $c:=$ cmin to $\mathrm{N}$ do \\ compute $\mathrm{P}_{\mathrm{w}}(\mathrm{C}, \mathrm{N}-\mathrm{C})$ by using "Algorithm 1 " compute $\mathrm{P}_{\mathrm{d}}(\mathrm{C}, \mathrm{N}-\mathrm{C})$ by using "Algorithm 1"}

To work out with some practical examples, we tested Algorithms 2 and 3 for $A_{1}=90, A_{2}=10, P_{w 0}=10^{-3}, P_{d 0}=10^{-4}$ and different values of $N$. For instance, for $N=145$ Algorithm 2 yielded $c \min =134$, that is, $c \in[134,145]$ as the feasible region for the waiting probability. Then, we applied Algorithm 3 to evaluate both probabilities along to the latter interval, and observed that in all cases the dropping probability was also falling below its upper bound. So definitely $c \in[134,145]$ is the final feasible region.

Something slightly different happened when $N=138$. In this case, the resulting feasible region for the waiting probability was [134,138], but the values obtained for the dropping probability by applying Algorithm 3 restricted this interval to [134,137].

In general, by varying $N$ between 135 and 150, we obtained a constant value of 134 for $\mathrm{cmin}$, and a quasi linear increase of the feasible region with cmax $\cong N$.

Also, we conclude from all experiments that usually the dropping probability either does not cause severe restriction on the feasible region of the waiting probability, or it cancels it completely. The reason is that the dropping probability decays very slowly as the number of common channels decreases while $N$ is fixed, and thus typically this probability falls almost completely below or above its prefixed upper bound.

\section{Conclusions}

In this paper, we have proposed an alternative architecture for a single cell in a LEO satellite cellular network aimed to support voice communications, and analyzed its performance. Based on a Markovian characterization of the model, we have derived exact closed form expressions for the waiting and dropping probabilities of new and handoff arrivals respectively, in terms of a system parameter and the Erlang-B formula. These expressions allow for the development of an efficient algorithm to compute both probabilities, as well as for the formulation and efficient implementation of a multiobjective optimization problem in the context of network planning.

Further investigation can focus on different problems:

- First, other multiobjective optimization problems can be formulated to account for alternative design requirements.

- Second, the analysis performed in this paper can be extended to more realistic scenarios consisting of networks of cells. In fact, when dealing with a network of cells, the handoff arrival rate should be determined as a function of the new arrival rate, and call blocking probabilities do not longer depend only on the availability of channels at the originating cell, but also on the availability of channels at the intersatellite links and the destination cell. Some related works can be found in [18-20]. 


\section{References}

1. Hong, D., Rappaport, S. S.: Traffic Model and Performance Analysis for Cellular Mobile Radio Telephone Systems with Prioritized and Nonprioritized Handoff Procedures. IEEE Transactions on Vehicular Technology, Vol. VT-35, No. 3 (1986) 77-92.

2. Haring, G., Marie, R., Puigjaner, R., Trivedi, K.: Loss Formulas and Their Application to Optimization for Cellular Networks. IEEE Transactions on Vehicular Technology, Vol. 50, No. 3 (2001) 664-673.

3. McMillan, D.: Delay Analysis of a Cellular Mobile Priority Queueing System. IEEE/ACM Transactions on Networking, Vol. 3, No. 3 (1995) 310-319.

4. Sato, T., Mori, M.: An Application of the Lumping Method to a Loss System with Two Types of Customers. Journal of the Operations Research Society of Japan, Vol. 26, No. 1 (1983) 51-59.

5. Kawashima, K.: An Approximation of a Loss System with Two Heterogeneous Types of Calls. Journal of the Operation Research Society of Japan, Vol. 28, No. 2 (1985) 163-176.

6. Yue, W., Matsumoto, Y.: Performance Analysis of Multi-Channel and Multi-Traffic on Wireless Communication Networks. Kluwer Academic Publishers, Boston (2002).

7. Gavish, B.: LEO/MEO Systems - Global Mobile Communication Systems. Telecommunication Systems, Vol. 8, pp. 99-141, 1997.

8. Stallings, W.: Wireless Communications and Networks. Prentice-Hall, 2002.

9. Dimitrijevic, D. D., Vuceti, J.: Design and Performance Analysis of the Algorithms for Channel Allocation in Cellular Networks. IEEE Transactions on Vehicular Technology, Vol. 42, No. 4, November 1993.

10. Boukhatem, L., Beylot, A. L., Gaïti, D., Pujolle, G.: Performance Analysis of Dynamic and Fixed Channel Allocation Techniques in a LEO Constellation with an Earth-Fixed Cell System. Globecom'00, San Francisco, November 2002.

11. Boukhatem, L., Beylot, A. L., Gaiti, D., Pujolle, G.: Channel Assignment with Handover Queuing in LEO Satellite Systems based on an "Earth-Fixed Cell" Coverage. ITC 17, Salvador da Bahia (Brazil), December 2001.

12. Del Re, E., Fantacci, R., Giambene, G.: Efficient Dynamic Channel Allocation Techniques with Handover Queuing for Mobile Satellite Networks. IEEE Journal on Selected Areas in Communications, Vol. 13, No. 2, February 1995.

13. Del Re, E., Fantacci, R., Giambene, G.: Handover Queuing Strategies with Dynamic and Fixed Channel Allocation Techniques in Low Earth Orbit Mobile Satellite Systems. IEEE Transactions on Communications, Vol. 47, No. 21, January 1999.

14. Ruiz, G., Doumi, T. L., Gardiner, J. G.: Teletraffic Analysis and Simulation of Mobile Satellite Systems. IEEE Transactions on Vehicular Technology, Vol. 47, No. 1, February 1998.

15. Guérin, R.: Queueing-Blocking System with Two Arrival Streams and Guard Channels. IEEE Transactions on Communications, Vol. 36, No. 2, February 1988.

16. Harrison, P. G., Patel, N. M.: Performance Modelling of Communication Networks and Computer Architectures. Addison-Wesley (1993).

17. Akimaru, H., Kawashima, K.: Teletraffic. Theory and Applications. Springer-Verlag, Germany (1993).

18. Zaim, A. H., Rouskas, G. N., Perros, H. G.: Computing Call-Blocking Probabilities in LEO Satellite Networks: The Single-Orbit Case. IEEE Transactions on Vehicular Technology, Vol. 51, No. 2, March 2002.

19. Zaim, A. H., Perros, H. G., Rouskas, G. N.: Computing Call-Blocking Probabilities in LEO Satellite Constellations. IEEE Transactions on Vehicular Technology, Vol. 52, No. 3, May 2003.

20. Ganz, A. et al.: Performance Study of Low Earth Orbit Satellite Systems. IEEE Transactions on Communications, Vol. 42, 1994. 